\title{
The Recent Advances of Fluorescent Sensors Based on Molecularly Imprinted Fluorescent Nanoparticles for Pharmaceutical Analysis*
}

\author{
Yi-fan WANG, Meng-meng PAN, Xu YU\#, Li XU\# \\ School of Pharmacy, Tongji Medical College, Huazhong University of Science and Technology, Wuhan 430030, China
}

(C) Huazhong University of Science and Technology 2020

\begin{abstract}
Summary: Fluorescent nanoparticles have good chemical stability and photostability, controllable optical properties and larger stokes shift. In light of their designability and functionability, the fluorescent nanoparticles are widely used as the fluorescent probes for diverse applications. To enhance the sensitivity and selectivity, the combination of the fluorescent nanoparticles with the molecularly imprinted polymer, i.e. molecularly imprinted fluorescent nanoparticles (MIFN), was an effective way. The sensor based on MIFN (the MIFN sensor) could be more compatible with the complex sample matrix, which was especially widely adopted in medical and biological analysis. In this mini-review, the construction method, detective mechanism and types of MIFN sensors are elaborated. The current applications of MIFN sensors in pharmaceutical analysis, including pesticides/herbicide, veterinary drugs/drugs residues and human related proteins, are highlighted based on the literature in the recent three years. Finally, the research prospect and development trend of the MIFN sensor are forecasted.
\end{abstract}

Key words: fluorescent sensor; molecularly imprinted polymer; pharmaceutical analysis; fluorescent nanoparticles

Fluorescence analysis, based on the fluorescence change reflecting the properties of the substances, is a broadly-used method for qualitative or quantitative analysis. Fluorescence analysis is superior to other instrumental analysis, e.g. gas chromatography, high performance liquid chromatography, capillary electrophoresis and so on, in terms of its low cost, simple operation, satisfactory sensitivity, less required equipment and fast detection speed. However, since few substances possess fluorescence property in nature, the direct fluorescence analysis of the target analytes is relatively seldom applied. Thus, some strategies are taken to render the analytes with fluorescent properties by derivatization ${ }^{[1,2]}$ and dye labeling ${ }^{[3,4]}$, etc. Recently, a more prevalent way relies on the fluorescent probe, by which the target analytes could be indirectly qualitatively or quantitatively analyzed by fluorescence change of the fluorescent probe.

Fluorescent probes are developed based on

Yi-fan WANG, E-mail: 524722013@qq.com

\#Corresponding authors, Xu YU, E-mail: xuyu@hust.edu.cn; Li XU, E-mail: xuyu@hust.edu.cn

${ }^{*}$ This work is supported by the National Natural Science Foundation of China (No. 21804105) and by the Fundamental Research Funds for the Central Universities (No.5003515037) and is partially supported by the Huazhong University of Science and Technology Start-up Fund to Xu YU. fluorescent organic molecules ${ }^{[5-7]}$ (e.g. commercial fluorescent dyes and homemade fluorescent molecules, etc.), or fluorescent nanoparticles ${ }^{[8-10]}$ (e.g. quantum dots, rare earth nanomaterials, etc.). Compared to fluorescent organic molecules, fluorescent nanoparticles are supposed to have better chemical stability and photostability (less or no photobleaching), controllable optical properties and larger stokes shift. Particularly, fluorescent nanoparticles can be deliberately designed for diverse applications by adjusting the preparation conditions or through the post modification. Also, they could have some reactivity, which would be appreciated for the effective construction of the fluorescent probes in different modes.

For the complex samples, the fluorescence analytical performance may be compromised, as a result of the negative sample matrix effect. Therefore, how to enhance the selectivity and sensitivity of fluorescence analysis and reduce the sample matrix influence remains as one bottleneck. Molecular imprinting technology, derived from the antigen-antibody theory in immunology, is well known for simulating the specific binding of antigen-antibody in nature, and adopted to prepare molecularly imprinted polymers (MIPs) which can specifically recognize template molecules or analogues ${ }^{[11]}$. Owing to their designability, specific recognition and wide practicability, MIPs are 
broadly used in sensors, chromatographic analysis, sample pretreatment and other fields ${ }^{[12-14]}$. Recently, the combination of MIP with fluorescent nanoparticles, i.e. molecularly imprinted fluorescent nanoparticles (MIFN), won increasing popularity. The sensor based on MIFN (MIFN sensor) would further enhance the detection selectivity and sensitivity of fluorescent analysis, and be more compatible with the complex sample matrix, which was especially appreciated in medical and biological analysis ${ }^{[15]}$.

Pharmaceutical analysis covers drug quality control, clinical pharmacy, traditional Chinese medicine and natural drug analysis, drug metabolism analysis, forensic toxicology analysis, stimulant detection and pharmaceutical preparation analysis, etc., which plays an irreplaceable role in the drug development, drug safety and drug effectiveness, and thus the precise medicine. The development of pharmaceutical analysis technologies is directed to the high sensitivity, high selectivity, high convenience and fast speed. The MIFN sensor possessed these characteristics, and found some applications in this filed. In this mini-review, the construction method, detective mechanism and types of MIFN sensors are elaborated. The current applications of MIFN sensors in pharmaceutical analysis, including pesticides/herbicide, veterinary drugs/drugs residues and human related proteins, are highlighted based on the recent three years' literature. Finally, the research prospect and development trend of MIFN sensors are forecasted.

\section{CONSTRUCTION OF THE MIFN SENSOR}

The MIFN sensor was composed with the fluorescent signal source and MIP specific recognition unit. By the assistance of MIP, the target analyte would be specifically recognized, and then interact with the fluorescent signal source to induce the fluorescence change, so that the target analyte could be quantified by monitoring the fluorescence intensity. The MIFN sensor based on fluorescent unit and MIP unit shows its advantages, such as excellent selectivity, good stability and easy operating. In this section, fluorescent nanoparticles to generate the MIFN sensor were summarized. In addition, preparation and detection mechanism of the MIFN sensor would be elaborated.

\subsection{Fluorescent Nanoparticles to Generate the MIFN Sensor}

In general, four types of fluorescent nanoparticles, including quantum dots (QDs) and carbon dots (CDs), rare earth materials, noble-metal nanoclusters (NCs) and metal-organic frameworks (MOFs), were predominantly employed as the fluorescence signal sources for the MIFN. The comparison of their advantages and disadvantages are summarized in table 1. The preparation of these materials was not the focus of this review, and elaborative related reviews could be referenced elsewhere ${ }^{[16-19]}$.

So far, the QDs/CDs were the most widely used fluorescence signal sources to construct the MIFN sensor, owing to their high luminous efficiency, optical stability and narrow emission spectrum. Recently, CDs were particularly appreciated as the fluorescence signal source for their environmental friendliness and nontoxicity while retaining the advantages of heavy metal QDs. Additionally, thanks to the narrow luminescence spectrum, large stokes shift (for lanthanide complexes) and possible upconversion property, rare earth fluorescent materials could be another good choice for the MIFN sensor; however, the application of this type of nanoparticles was not that much in pharmaceutical analysis. For the other two fluorescent nanoparticles, NCs and MOFs, the applications were further less, which may be due to the high cost and low quantum yields of these nanoparticles. Anyway, in the future work, the exploration of appropriate fluorescent nanoparticles for the MIFN sensor should be highly expected for more diverse pharmaceutical analysis.

Table 1 Summary of characteristics of fluorescent nanoparticles for the MIFN sensor

\begin{tabular}{|c|c|c|}
\hline $\begin{array}{l}\text { Type of fluorescent } \\
\text { signal sources }\end{array}$ & Advantages & Disadvantages \\
\hline QDs \& CDs & $\begin{array}{l}\text { QDs: good light stability, high quantum yield, and the } \\
\text { emission spectrum can be controlled by changing their } \\
\text { size and chemical composition. } \\
\text { CDs: besides the above characteristics of QDs, non- } \\
\text { toxic and eco-friendly, and the sources of raw materials } \\
\text { are extensive and the cost is low. }\end{array}$ & $\begin{array}{l}\text { There are toxicity problems for heavy metal QDs. } \\
\text { For CDs, further research is needed to make clear } \\
\text { the structure, luminescence mechanism and so on. }\end{array}$ \\
\hline Rare earth materials & $\begin{array}{l}\text { Strong light absorption, high conversion rate, wide } \\
\text { emission wavelength distribution region and good } \\
\text { stability. }\end{array}$ & $\begin{array}{l}\text { The cost is higher than other fluorescent } \\
\text { nanoparticles. }\end{array}$ \\
\hline Nobel-metal NCs & $\begin{array}{l}\text { The synthesis condition is mild, the light stability and } \\
\text { the biocompatibility is good, and fluorescence properties } \\
\text { are controllable. }\end{array}$ & $\begin{array}{l}\text { Easy to gather, chemical stability and quantum } \\
\text { yields are relatively poor, the cost is high. }\end{array}$ \\
\hline MOFs & $\begin{array}{l}\text { Predictable structure, diverse functions, and high } \\
\text { specific surface area. }\end{array}$ & $\begin{array}{l}\text { Luminescent MOFs materials usually need to be } \\
\text { doped with rare earth, transition elements, or other } \\
\text { metal ions to form heteronuclear MOFs materials. }\end{array}$ \\
\hline
\end{tabular}




\subsection{Preparation of the MIFN Sensor}

Generally, three steps were involved to prepare the MIFN sensor, including: (1) preassembly of fluorescent nanoparticles, the functional monomer and the template molecule in a solvent. The fluorescent nanoparticles could be directly used, or used after proper modification. The modification could be achieved by silylation ${ }^{[21,22]}$, or being treated with oleic acid ${ }^{[23]}$, polyethyleneimine ${ }^{[24]}$, or polyethyleneglycol ${ }^{[25,26]}$, aiming to provide functional groups or compatibility with synthetic medium for better embedding the fluorescent nanoparticles in the MIP; (2) Polymerization. The crosslinking agent was added to the pre-assembled system, and the polymerization proceeded to form a highly-crosslinked polymer triggered by the initiator or catalyzed by the catalyst; (3) Removal of the template. The template molecule was removed from the polymer mostly via the solvent dissolving approach, so as to leave stereo cavities in the polymer matching the size and shape of the template molecule. Thanks to the active groups provided by functional monomers in the cavities, which were precisely arranged and complementary to the functional groups of the template molecules, the imprinted polymers had a specific "memory", i.e. the specific binding sites and recognition ability, towards the template molecules or analogues.

The recognition ability of the MIFN sensor was largely determined by the MIP, which was influenced by various factors ${ }^{[1-15]}$, such as template molecules, functional monomers, crosslinking agents, type and dosage of solvent, polymerization temperature and time, and initiation mode, etc. These factors should be optimized case by case. Especially, template molecules, functional monomers and crosslinking agents were the crucial determining factors to the recognition ability of the MIFN sensor ${ }^{[15]}$. Template molecules were usually the target molecules to be detected, and structural analogues were also selected as dummy template molecules in some cases. An ideal template molecule should contain active groups which interacted with the functional monomers, but the molecule itself was relatively stable and resistant to prevent or participate in the polymerization reaction. Functional monomers were molecules which provided covalent or non-covalent binding with template molecules and copolymerized with crosslinkers, which had important effects on the availability of binding sites and the selectivity. The role of crosslinking agents was to fix functional groups of functional monomers around the template molecules, so as to ensure that cavities complementary to the template molecules on the shape and functional groups were created to form highly-crosslinked and rigid imprinted polymers after removing the template molecules. It is worth mentioning that the solvent in the preparation was not only as a solvent media for polymerization, but also as a porogen for the MIFN.

For the preparation of the MIFN sensor, three synthetic methods were frequently used, i.e. sol-gel method, free radical polymerization, and reverse microemulsion method, in that order. The preparation of the MIFN sensor is depicted in fig. 1.

The sol-gel approach was the most oftenused method, mainly to generate silica-based MIP. 3-Aminopropyl triethoxysilane (APTES), tetraethoxysilane (TEOS) and ethanol were usually the monomer, crosslinking agent and solvent, respectively. APTES first interacted with the template and the fluorescent nanoparticles in a rational ratio, followed

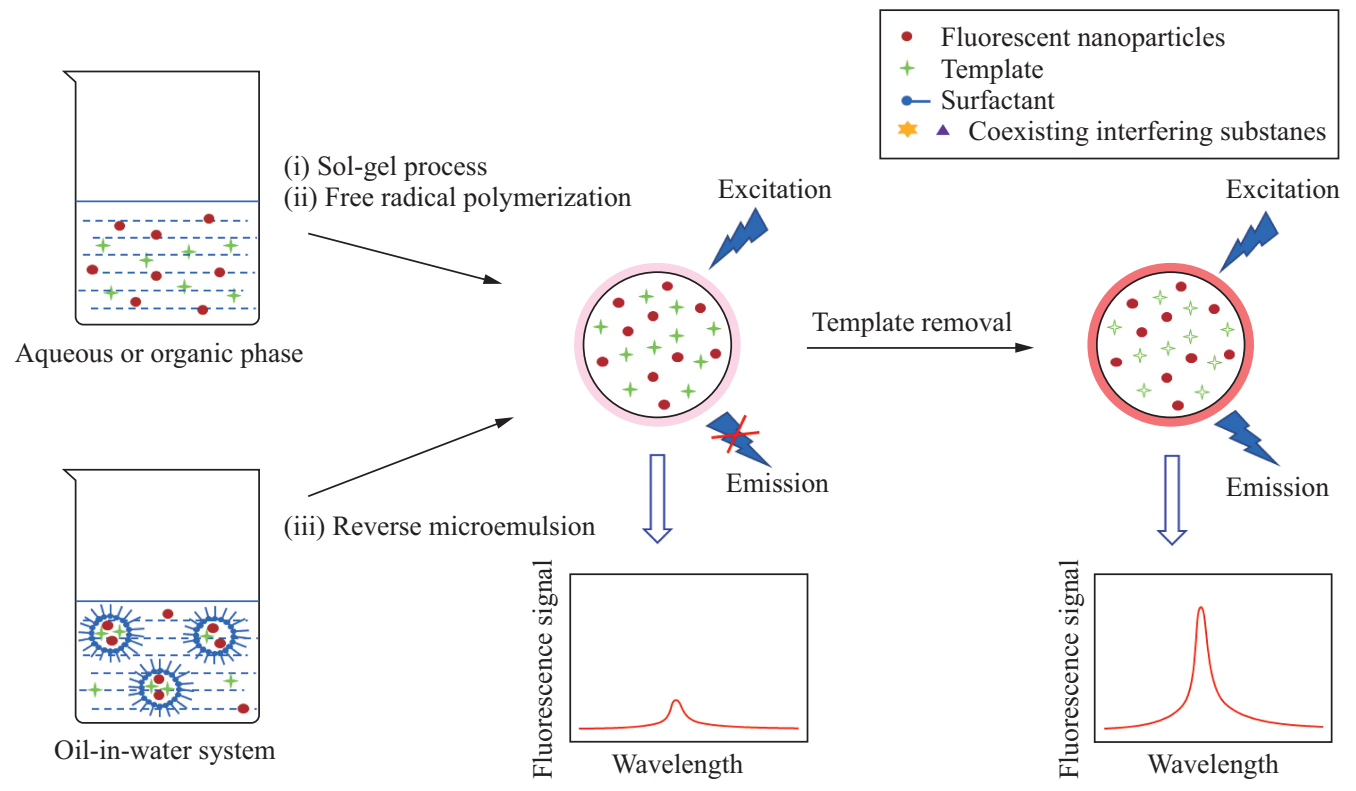

Fig. 1 Schematic diagram of the synthesis of the MIFN sensor 
by the addition of TEOS and the catalyst, with the hydrolysis and condensation proceeding. The solgel approach could proceed in mild and controllable synthetic condition, even at room temperature. The product uniformity was good, and morphology could be controlled by fine-tuning the type and concentration of silane, the $\mathrm{pH}$ of reaction system and so on. The ratio of the functional monomer to the crosslinker was mostly found to range from $1 / 1$ to $1 / 20$, which determined the property of the MIP. The excessive functional monomer or crosslinker would cause the MIP layer to be thick to deteriorate the luminous efficiency and responsive speed of the fluorescent probe, which was detrimental to the fluorescent detection performance; contrarily, the MIP layer would be insufficient if the amount of monomer and crosslinker was insufficient, which led to a decrease in sensitivity and selectivity. The amount of the catalyst, reaction $\mathrm{pH}$ and temperature would affect the speed of the sol-gel reaction, thus influencing the microstructure of the final material. In one case, Guo et al[ ${ }^{[27]}$ synthesized the MIFN sensor for specific measurement of aflatoxin $\mathrm{B}_{1}$, using $\mathrm{CdTe}$ QDs, 5,7-dimethoxycoumarin, APTES and TEOS as fluorescent sources, dummy template, functional monomer and cross-linker, respectively. It was found that the best molar ratio of 5,7-dimethoxycoumarin to APTES to TEOS was $4: 20: 15$, resulting in the highest sensitivity. In another study, the dual functional monomers, APTES and 2-(4-chlorosulfonylphenyl) ethyl-trimethoxysilane in a molar ratio of $1 / 1$ were found to be more effective than single monomer APTES, in terms of higher quenching efficiency and imprinted factor ${ }^{[28]}$.

Apart from the above sol-gel method, the reverse microemulsion was another approach to generate the silica-based MIPs for the MIFN sensor ${ }^{[10,29-33]}$. The reverse microemulsion was an oil-in-water thermodynamically stable micelle system composed of the oil phase, water phase and reverse micelle. The oil phase was a continuous phase, and the water phase was inside the reverse micelle formed by the surfactant, which was the reactor for the synthesis of nanomaterials. It is worth mentioning that the surfactant played essential roles in the reverse microemulsion process, including reducing interfacial tension, determining the type of microemulsion, resulting in interfacial tension gradient and Gibbs-Marangnoi effects, causing electrostatic effects and steric repulsion effects, and avoiding the formation of liquid crystalline phases ${ }^{[34]}$. Usually, cyclohexane and Trion X-100 were selected as the oil phase and surfactant for preparation of the MIFN sensor with the volume ratio of about $4.2^{[29-33]}$. Similar to the sol-gel method, the most commonly used monomer and crosslinking agent were APTES and TEOS for the reverse microemulsion, respectively. Also, the reaction conditions of reverse microemulsion were mild, and the products were generated with controllable morphology and uniformity. Compared to the sol-gel method, the reverse microemulsion method often resulted in smaller particle size. In addition, since the sol-gel process proceeded in the aqueous or ethanol media while the inverse microemulsion method included both the water phase and oil microemulsion phase, it was a critial challenge to select the appropriate synthetic method to ensure the performance of the MIFN sensor according to the solubility of the target analytes. For example, Guo et $a l^{[27]}$ compared the sol-gel process and reverse microemulsion to prepare the 5,7-dimethoxycoumarin imprinted MIFN sensor. The recognition effect of the probe prepared by reverse microemulsion was poor. The reason for this observation was that the QDs and the monomers were dispersed in the aqueous phase, while the template molecules were dispersed in the oil phase, resulting in low efficiency of MIPs.

Free radical polymerization was based on the polymerization reaction of monomers containing unsaturated double bonds initiated by free radicals. As summarized in table 2, the commonly-used functional monomers included methacrylic acid (MAA), acrylamide (AAm) and vinylpyridine (VP), while the commonly-used crosslinking agents were ethylene dimethacrylate (EGDMA), trihydroxymethylacrylate (TRIM), diethylbenzene (DVB), and N,N-methylenebisacryamide (MBA). Moreover, low polar solvents, e.g. acetonitrile, dichloromethane, chloroform, etc., played dual roles as the reaction media and porogen. Additionally, the free radical polymerization reaction normally needs to be initiated by the heat or light. Heating initiation required a certain high temperature depending on the initiator; besides, the initiator was extra introduced to the polymerization system, leading to side effects in some cases. Unlike the heating initiation, the photo initiation was performed without initiators, but with less side reactions and energy consumption; however, it was less often used than the thermal initiation method. The reason may be that the light used for initiation may excite the fluorescent nanoparticles to produce fluorescence, which may interfere with the light and be unfavorable to the polymerization reaction. For example, Geng et al ${ }^{[35]}$ coated MIPs on 3-mercapto propionic acid modified CdSe QDs via free radical polymerization with MAA and thiol modified aptamer as the functional monomers, MBA as crosslinker and ammonium persulfate and $\mathrm{N}, \mathrm{N}, \mathrm{N}^{\prime}, \mathrm{N}^{\prime}-$ tetramethylethylethylenediamine as initiator at $40^{\circ} \mathrm{C}$. The ratio of template, aptamers and MAA was optimized as 1:1:5. The resulting sensor was applied to specifically detect the kanamycin in tap and lake water, milk and urine samples. Li et al ${ }^{[36]}$ synthesized N, S codoped CDs and gold nanoparticles firstly. They were conjugated onto the surface of an indium tin oxide glass 


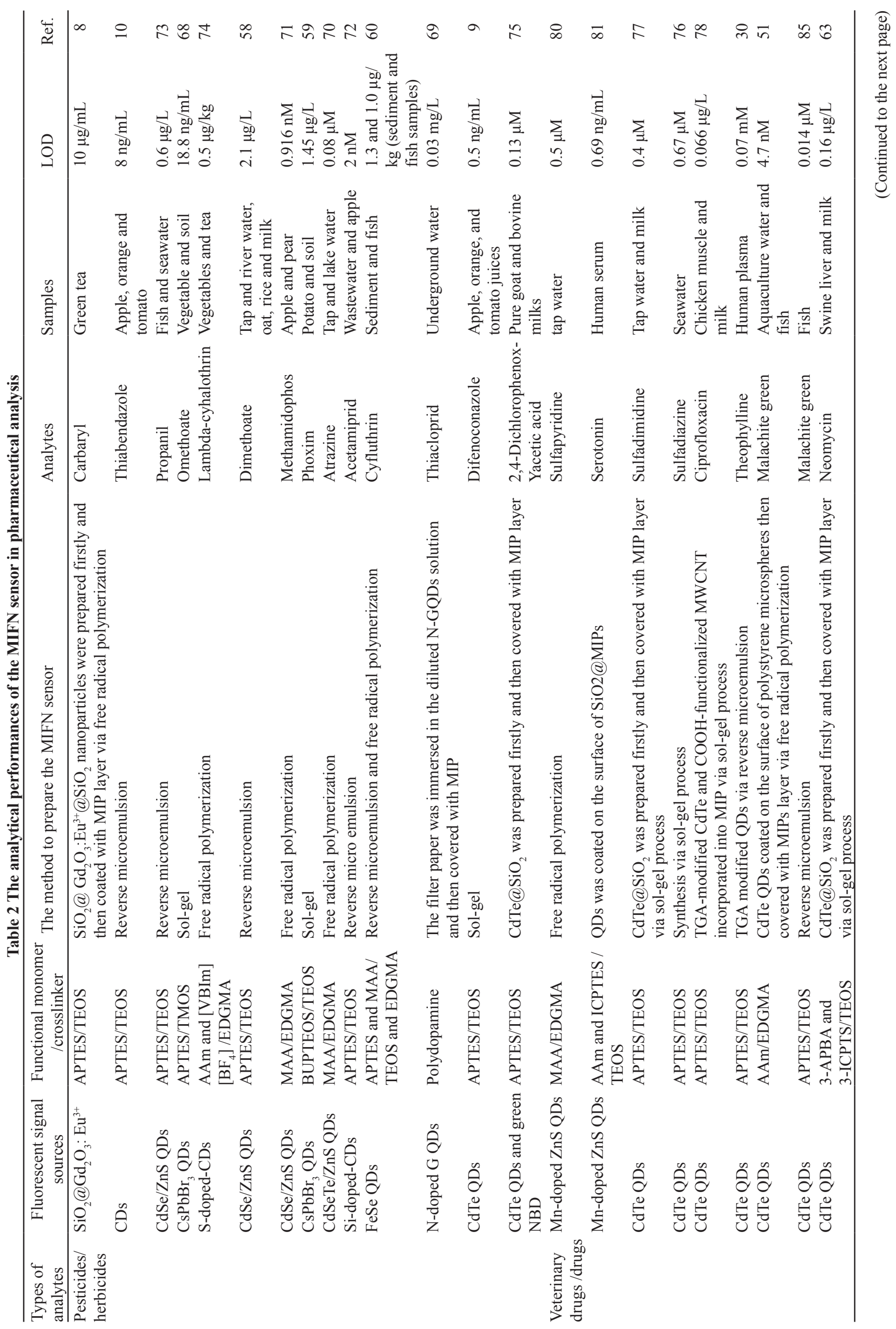




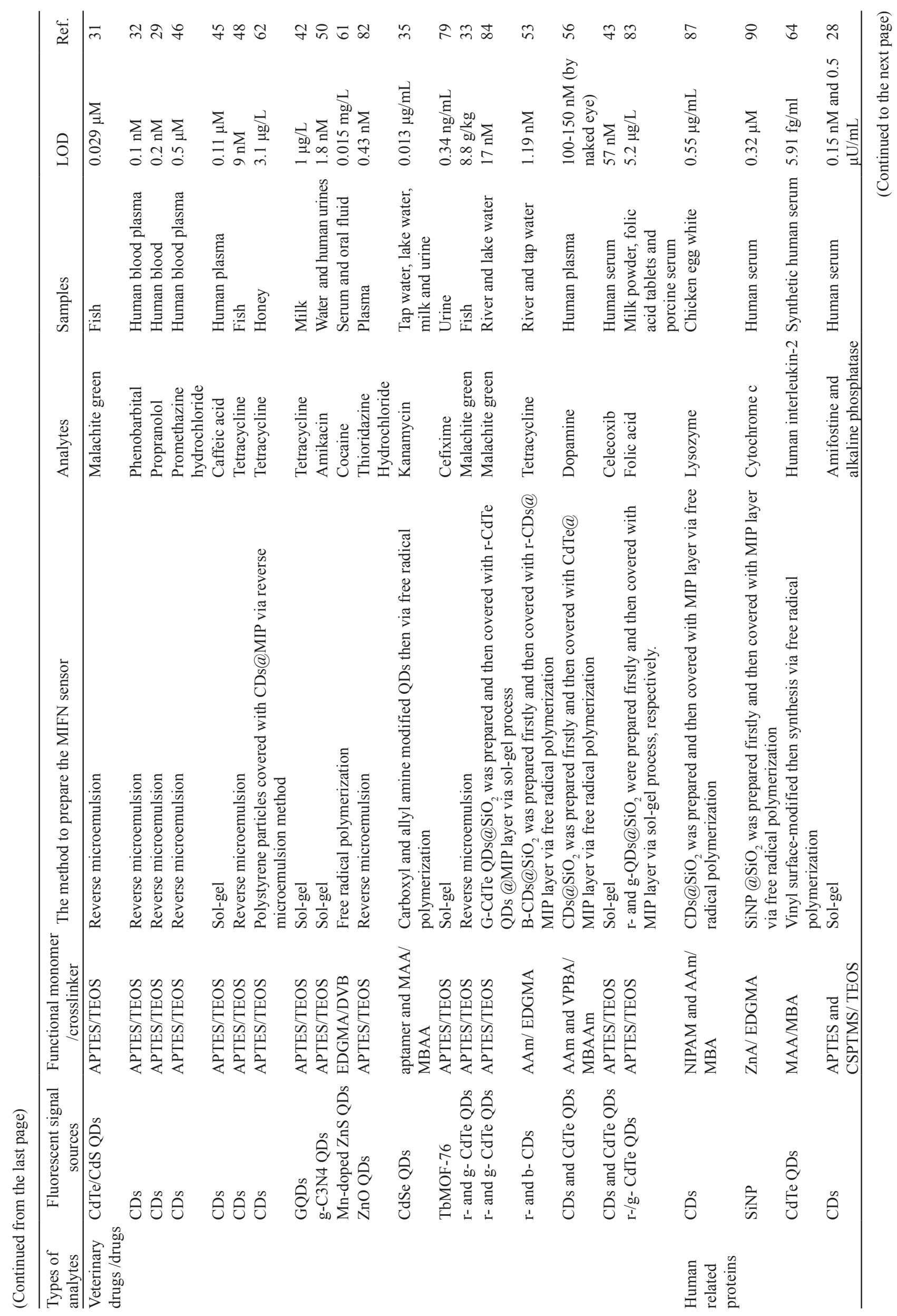




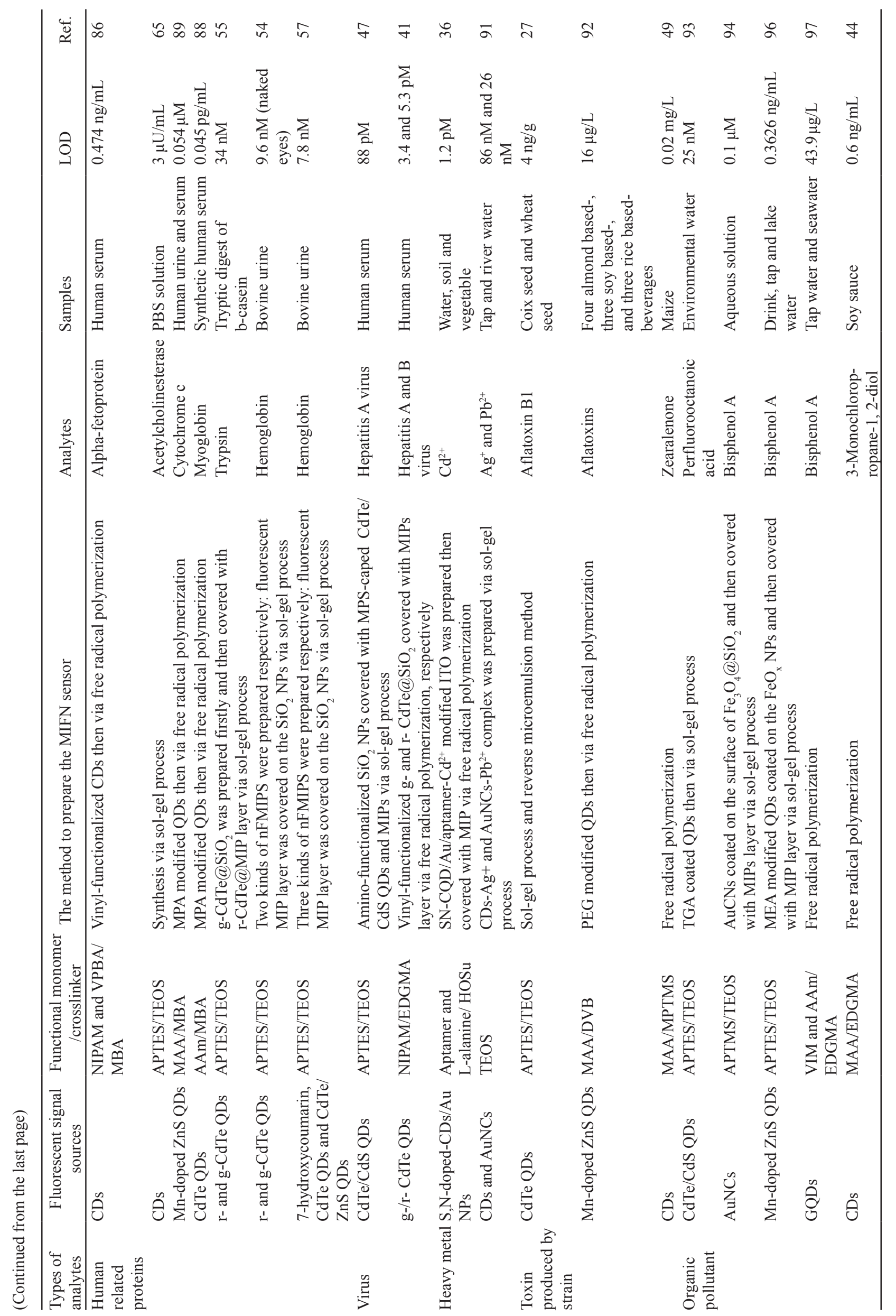


electrode, followed by the assembly of aptamer- $\mathrm{Cd}^{2+}$ complexes via an Au-S bond. The MIP layer was then produced on the surface of this assembly with L-alanine as a functional monomer and N-hydroxysuccinimide as a crosslinking agent by ultraviolet irradiation. After removing the template, the obtained sensor was applied to detect $\mathrm{Cd}^{2+}$ in water, soil and vegetable samples.

\section{DETECTION MECHANISM OF THE MIFN SENSOR}

In the MIFN sensor, the nano-fluorescent signal source played a role as fluorescent response. Although some fluorescent nanomaterials, such as CDs, had certain selectivity, which were reported to directly detect $\mathrm{Fe}^{2+[37,38]}$ and $\mathrm{Hg}^{2+[39,40]}$, etc., most fluorescent nanomaterials had limited selectivity in applications. Hence, MIP was constructed specifically to further improve the selectivity and sensitivity of the sensor as a result of its certain adsorption capacity. It should be noted that the thickness of the MIP layer needs to be reasonably controlled. The thick MIP layer might weaken the fluorescence and increase the difficulty of the template removal; while the thin MIP layer would provide limited identification sites, resulting in unsatisfactory selectivity and sensitivity.

The basis of the MIFN sensor detection can be classified to the fluorescence quenching and fluorescence enhancement. Fluorescence quenching of the MIFN sensor caused by target analyte was the basis of the mostly reported MIFN sensor so far. Fluorescence quenching can be divided into static or dynamic quenching as a whole $\mathrm{e}^{[41,42]}$, and the specific quenching mechanism included photoinduced electron transfer ${ }^{[43-45]}$, energy resonance transfer ${ }^{[46,47]}$ and inner filter effect ${ }^{[48]}$, etc. The mechanism was determined by the characteristics of specific nano-fluorescent signal sources and target analytes. For example, the mechanism belonged to dynamic quenching or static quenching can be judged by fluorescence lifetime, temperature effect, variation of fluorescence material absorption spectrum.

Fluorescence enhancement of the MIFN sensor caused by the target analyte was far less used than the fluorescence quenching. Energy resonance transfer was mainly responsible for the fluorescence enhancement ${ }^{[8,35]}$, reflected by the large overlap of the wavelength between the fluorescence peak of target analytes and the ultraviolet absorption peak of the sensor.

\section{TYPES OF THE MIFN SENSOR}

According to the different fluorescence emission peaks, the MIFN sensor can be categorized into two modes: single-emitting mode and multi-emitting mode.
The sensing mechanism of these two types of MIFN sensors is depicted in fig. 2.

\subsection{Single-emitting MIFN Sensor}

The single-emitting MIFN sensor contains only one fluorescence signal source, which can be any one of the above fluorescence nanoparticles in Section 2.1. In this type of MIFN sensor, the target analytes can be selectively captured by MIPs, and then interact with the fluorescent signal source, resulting in the change of its fluorescence intensity, to achieve the purpose of qualitative and quantitative analysis ${ }^{[4-51]}$, as depicted in fig. 2A.

To further enhance the detection sensitivity, the amplification strategy could be adopted. Through the self-assembly of CdTe QDs onto polystyrene microspheres via electrostatic interaction followed by covering with MIP, the fluorescence quenching of CdTe QDs by the target analyte was amplified, due to the "local concentration ability" of the polystyrene microspheres. The method was successfully used for specific detection of malachite green in water and fish samples with a low LOD of $4.7 \mathrm{nM}^{[51]}$.

Although there was only one emission peak in the single-emitting MIFN sensor, the ratiometric fluorescence quantitation was still expected when the target analyte itself had fluorescence. Fu et al ${ }^{[52]}$ used blue-emitting CDs decorated $\mathrm{Fe}_{3} \mathrm{O}_{4}$ nanoparticles for specific detection of red-emitting phycoerythrin. The fluorescence of CDs was gradually quenched with the increasing concentration of phycoerythrin, due to the inner filter effect since the large overlap existed between the UV-vis absorption spectrum of phycoerythrin and the fluorescence emission spectrum of the CDs; meanwhile, the fluorescence of phycoerythrin was increased. Thus, the fluorescence intensity ratio of CDs and phycoerythrin was used for quantitative analysis of phycoerythrin in the human serum sample, real seawater and river water samples with an obviously lowered LOD of $1.5 \mathrm{ng} \cdot \mathrm{mL}^{-1}$.

\subsection{Multi-emitting MIFN Sensor}

For the multi-emitting MIFN sensor, at least two or more emission peaks appeared, deriving from one or more fluorescent signal sources, once it was excited at the single particular wavelength. The fluorescence intensity at different emission wavelengths varied to different degrees after the target analytes were identified by the MIPs and interacted with the fluorescent signal sources; thus, the relationship of ratio of fluorescence intensity at different wavelengths with the concentration of target analytes could be expected for quantification, which was the ratiometric fluorescence sensor ${ }^{[53-55]}$, as depicted in fig. 2B. Compared with the single-emitting MIFN sensor, the multi-emitting fluorescent probe can enhance the dynamic response range, the accuracy and sensitivity of the measurement ${ }^{[43,53-57]}$.

As a general approach to construct ratiometric 
A: Single-emitting mode

(Taking fluorescence quenching as an example)
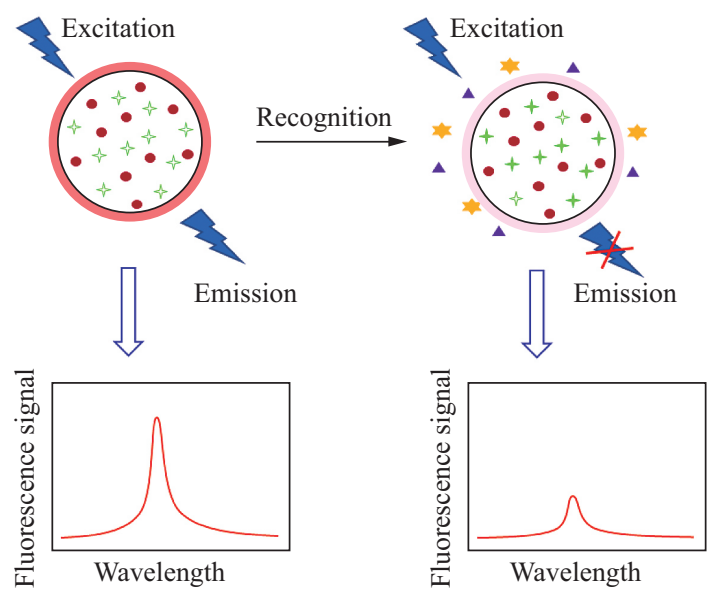

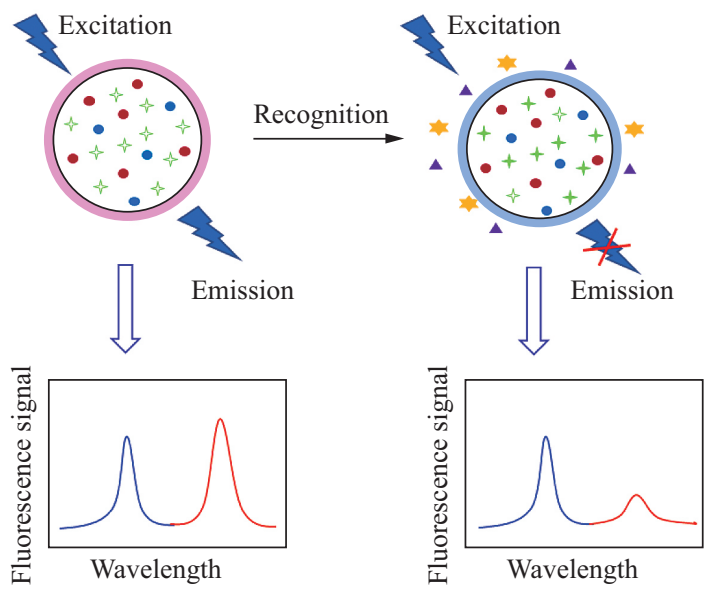

- Fluorescent nanoparticles

+ Template

* Coexisting interfering substanes

Fig. 2 The sensing mechanism of the MIFN sensor

A: The single-emitting MIFN sensor (Taking the red-emitting fluorescent nanoparticle as the fluorescent source, and fluorescence quenching as detection mechanism). B: The dual-emitting MIFN sensor (Taking both red- and blue-emitting fluorescent nanoparticles as fluorescent sources, and the target analyte quenched the red fluorescence more than the blue one).

fluorescence sensor, two different color emission fluorescence nanoparticles were adopted. For example, Liu et $a l^{[53]}$ synthesized red-emission CDs and blueemission CDs from the same natural carbon source of osmanthus fragrans leaves, but with different solvent extractions. The ratiometric fluorescence sensor was constructed with the red-emission CDs as responsive signal and the blue-emission CDs as reference signal. The blue-emission CDs were coated with a silica layer, effectively inhibiting their direct interaction with the target analyte (tetracycline), thus as reference signal. Afterwards, the MIPs layer toward tetracycline was constructed on the red-emission CDs and the silica coated blue-emission CDs as the core. In the presence of tetracycline, the fluorescence of red-emission CDs was quickly quenched while the fluorescence of blueemission CDs remained unchanged. The sensor was applied for highly selective and sensitive detection of tetracycline in local river water and tap water with an LOD of $1.19 \mathrm{nmol} / \mathrm{L}$ and linearity range of $0-50$ $\mathrm{nmol} / \mathrm{L}$.

Another appealing merit of the multi-emitting MIFN sensor was its colorimetric fluorescence ability. When the target analytes bound with the MIFN sensor, the fluorescence intensity at different wavelengths would vary to different degrees; as the fluorescence color at different wavelengths was different, the fluorescence intensity change would lead to the color change of the sensor. In light of this color change, colorimetric fluorescence could be established so that the target was detected semi-quantitatively by the naked eye or precisely quantitatively in combination with portable electronic devices such as smartphones. This approach was especially appreciated for on-site detection or point-of-care testing. Wang et al ${ }^{[56]}$ used blue-light CDs as the reference signal source and redlight CdTe QDs as fluorescent responsive signal source to prepare the ratiometric MIFN sensor for dopamine detection in the human plasma. The blue CDs had no response to the dopamine while the fluorescence of the red CdTe QDs was quenched by the dopamine, so the concentration of dopamine was accurately quantified by the fluorescence intensity ratio of the CDs to the QDs. Meanwhile, the quenching of red fluorescence caused the color transition from fuchsia to blue gradually, which made it possible to roughly judge the concentration of dopamine through the naked eye. Moreover, the concentration of dopamine could be also calculated by the smartphone with the RGB value analysis to avoid visual differences.

Recently, a ternary-emission fluorescence sensor was developed to widen the color change window ${ }^{[57]}$. The blue-/green-/red-emission bovine hemoglobin $(\mathrm{BHb})$ imprinted polymers were individually prepared. These three colored MIPs were mixed at a proper ratio for the multiplexed and visual detection of $\mathrm{BHb}$. Once interacting with $\mathrm{BHb}$ for $8 \mathrm{~min}$, the green and red MIPs exhibited weakened fluorescence, whereas the blue MIP had strengthened fluorescence; thus, the green-red-blue window was observed. The strategy 
was beneficial to enhance the determination accuracy of the $\mathrm{BHb}$ with the naked eyes.

\section{APPLICATION OF THE MIFN SENSORS IN PHARMACEUTICAL ANALYSIS}

The MIFN sensor possessed good selectivity, high sensitivity, simple and rapid operation, which was suitable for the detection of target analytes in the complex samples. In this section, the applications of MIFN sensors in pharmaceutical analysis were highlighted, as listed in table 2.

\subsection{Detection of the Pesticide/herbicide Residues}

The long-term and large-scale usage of pesticides and herbicides in agricultural product disrupts ecological balance, increases pesticide/herbicide residues in environment and related foodstuff, and thus endangers human health. Thanks to the high selectivity of MIPs, the MIFN sensor could reduce the steps of sample preparation prior to measurement and enable the simple and rapid detection of pesticides/herbicides from the complex environmental and food samples.

Yang et al[58] coated MIPs on CdSe/ZnS QDs using the modified reverse microemulsion with APTES and TEOS as functional monomer and crosslinker, respectively, for the detection of dimethoate. The determination of dimethoate by the prepared sensor was realized with satisfactory recoveries of $89.8 \%-98.0 \%$ in the spiked tap and river water, oat, rice and milk. Moreover, with the help of 96-well microplate, the highthroughput detection of dimethoate could be achieved.

In some cases, the functional monomer was deliberately designed for better specificity. Taking phoxim as the target, a new siloxane, i.e. N-(benzyl)N'-(3-(triethoxysilyl)propyl)urea, was designed and synthesized, to provide multiple functional groups to interact with the target ${ }^{[59]}$. By using N-(benzyl)-N'-(3(triethoxysilyl)propyl)urea as the functional monomer, $\mathrm{CsPbBr}_{3}$ QDs as fluorescent signal and TEOS as crosslinker, the obtained sensor was successfully used for selective detection of phoxim in potato and soil with the recoveries of $86.8 \%-98.2 \%$.

To enhance the selectivity, in Li's work ${ }^{[60]}$, both MAA and APTES were used as functional monomers, TEOS and EDGMA were used as crosslinkers to prepare the FeSe QDs embedded silica MIP by reverse microemulsion method. This sensor had high selectivity for the template, cyfluthrin, via multiple interactions including ionic interaction, shape selectivity and hydrogen bonding interaction. The LODs of cyfluthrin in sediment and fish samples were as low as $1.3 \mu \mathrm{g} / \mathrm{kg}$ and $1.0 \mu \mathrm{g} / \mathrm{kg}$, respectively.

\subsection{Detection of the Veterinary Drugs Residues}

Due to the improper use of veterinary drugs and excessive use of medical drugs in production, serious residues were produced in the food of animal origin, like animal muscles, milk and liver, etc., which posed a threat to human health. Based on high selectivity and good sensitivity, the MIFN sensor could efficiently identify and detect veterinary drugs in complex food and biological samples, achieving specific, simple and rapid detection.

Maria et $a l^{[61]}$ anchored a selective MIP for cocaine on the surface of polyethylene glycol modified Mndoped ZnS QDs. The fluorescence of the sensor was gradually quenched with the increasing concentration of the cocaine. Thus, a simple and low-cost method was established for assessing cocaine abuse. The LODs of the cocaine detection were $0.035 \mathrm{mg} / \mathrm{L}$ in oral fluid and $0.015 \mathrm{mg} / \mathrm{L}$ in serum, respectively.

Since the food and biological sample matrix may have the fluorescence to interfere the fluorescence detection, some strategies should be employed to reduce or eliminate the interference. In Li's study ${ }^{[62]}$, the target analyte, tetracycline, in diluted honey sample, was firstly adsorbed by the constructed sensor, owing to the good adsorption ability of MIP. Afterwards, the sensor adsorbing tetracycline was separated by centrifugation from honey sample and re-dispersed into phosphate buffer solution. In this manner, the auto-fluorescence of the honey sample which might interfer with the fluorescence signal of the sensor was avoided. The sensor was reusable with $96.5 \%$ fluorescence intensity of its original one after six recycles. The LOD of the sensor was as low as $3.1 \mu \mathrm{g} / \mathrm{L}$ and the recoveries ranged from $93 \%$ to $105 \%$.

Although the MIFN sensor was supposed to be directly used for the complex samples, the macromolecules may in some cases interfere with the detection and compromise the detection performance of the sensor. To address this problem, combining sample pretreatment with the detection process could be an alternative choice. To determine neomycin in pork, swine liver and kidney, fish meat and liver, chicken meat and kidney, and milk samples, the samples were firstly pre-treated by liquid-liquid extraction and solidphase extraction to isolate and concentrate neomycin, and then subjected to the MIFN sensor and HPLC-FLD analysis, respectively ${ }^{[63]}$. The results indicated that both the sensitivity and selectivity of the MIFN sensor were higher than those of HPLC-FLD.

\subsection{Detection of Human Related Proteins}

Protein is an important component of human cells and tissues, and the abnormal expression of protein is closely related to the occurrence and development of various diseases. For example, urine protein content effectively reflects the occurrence of gestational hypertension. Therefore, it is crucial to monitor protein content in the biological fluids. The commonly used methods for the protein detection include western blotting, enzyme-linked immunosorbent assay (ELISA), HPLC, GC, etc. The HPLC and GC methods 
required expensive instrument and skilled operators. Both western blotting and ELISA were carried out based on molecular recognition between antigens and antibodies with high sensitivity and good specificity. However, the preparation of antibodies was complicated and costly, and antibodies were very sensitive to the detection environment (such as temperature, $\mathrm{pH}$, etc.). As a compromise, good stability and low cost were the characteristic of the MIFN sensor, in addition to the high sensitivity and specific recognition. Hence, the MIFN sensor had a great application prospect for protein detection.

As the same synthetic approach to the small molecules, a MIFN sensor was designed by using CdTe QDs, human interleukin-2 (IL-2), MAA and MBA as fluorescent sources, template, functional monomer and crosslinker, respectively. This sensor recognized and determined IL-2 in synthetic human serum with an LOD of $5.91 \mathrm{fg} / \mathrm{mL}$, which was below the concentration levels of IL-2 with clinical interest for cancer diagnosis $(9.4-19.2 \mathrm{pg} / \mathrm{mL})^{[64]}$.

The visual detection of $\mathrm{BHb}$ was achieved by simply mixing the solution of green and red CdTe QDs, which were embedded in the core-shell structured MIPs and silica nanoparticles, respectively. The green QDs were selectively quenched by the BHb, while the fluorescence of the red QDs with silica shell kept stable to the $\mathrm{BHb}$ due to the protection of the silica layer. In this manner, a continuous obvious fluorescence color change from green to red could be observed by naked eyes, with the LOD of $9.6 \mathrm{nmol} / \mathrm{L}$. The applicability of this sensor was demonstrated by determining $\mathrm{BHb}$ in bovine urine samples with satisfactory recoveries from $95.7 \%$ to $101.5 \%{ }^{[54]}$.

It is worth mentioning that the MIFN sensor can be used for evaluation of inhibitor assay, when the target analyte is the enzyme. Zhao et a ${ }^{[65]}$ established the sensor sensitive to acetylcholinesterase (AChE) by the sol-gel process. The sensor was not only used to specifically detect AChE in PBS solution, but also to evaluate the effect of inhibitor, huperzine $\mathrm{A}$. The maximum inhibition rate and $\mathrm{IC}_{50}$ value of huperzine $\mathrm{A}$ was similar to previous reports.

\subsection{Detection of Virus}

The infectious viruses have caused hundreds of thousands of deaths each year ${ }^{[66]}$. To date, SARSCov-2, one kind of enveloped positive-sense RNA viruses, has infected more than 3000000 people worldwide and killed more than 200000 people ${ }^{[67]}$. The detection of the viruses has become a hot topic. Current methods for identification and quantification of the particular viruses, including polymerase chain reaction (PCR) and ELISA, etc., are often time consuming and expensive within special laboratories. As a high potential complementary method for virus detection, the MIFN sensors aroused various interests among the researchers owing to their time saving, low-cost and point-of-care testing properties.

Luo and coworkers developed several MIFN sensors for hepatitis A virus (HAV) and hepatitis B virus (HBV) detection ${ }^{[41,47]}$. With $\mathrm{CdTe} / \mathrm{CdS}$ QDs, HAV, APTES and TEOS as fluorescent source, template, functional monomer and crosslinker, respectively, the established sensor could specifically detect HAV in human serum with an LOD of 88 $\mathrm{pmol} / \mathrm{L}$ and recoveries of $96.7 \%-103.8 \%{ }^{[47]}$. Lately, the same team ${ }^{[41]}$ synthesized red and green CdTe QDs to construct the multi-emission MIFN sensor. The LOD for HAV was further lowered to $3.4 \mathrm{pmol} / \mathrm{L}$, and for HBV was $5.3 \mathrm{pmol} / \mathrm{L}$. Besides, visual detection of these two kinds of viruses was possibly based on the colorimetric fluorescence.

\subsection{Detection of Other Pollutants in Pharmaceutical Related Samples}

The other pollutants, e.g. heavy metal, toxin produced by strain, and organic pollutants were also detected by MIFN sensors in pharmaceutical related samples, as listed in table 2 .

\section{CONCLUSION AND PROSPECT}

The MIFN sensor combines the high selectivity of MIPs with the high sensitivity of the fluorescent nanoparticles, and converts molecular recognition into a readable fluorescent signal. The integration of specific identification and signal response units boosts the application range of the MIFN sensor, enabling the high sensitive detection of trace substances in complex matrices. Compared with other instrumental based analytical techniques, the MIFN sensor is easy to operate, fast to detect and has low cost, thus showing great potential application prospects.

The MIFN sensor provides an excellent method for the specific detection of target analytes in complex matrices, but this technique has not been fully developed and needs further exploration in pharmaceutical analysis. Firstly, the preparation process with the existing synthetic methods still has the disadvantages of long reaction time, heterogeneous particle size and non-uniform interaction sites. More precise controlling synthesis methods need to be developed. Secondly, for complex sample matrix, the anti-interference ability of the MIFN should be further enhanced to achieve the satisfactory selectivity and sensitivity. Last but not the least, the MIFN sensor for simultaneous detection of more than one target analytes is seriously lacking. This is an important development direction towards multiple analytes' detection.

In summary, with the application of the MIFN sensor in pharmaceutical analysis, preparation of more powerful MIFN sensor and strengthening of its combination with smart devices like smartphone to 
create new smart fluorescence rapid detection platforms still need further study. There is much room for the MIFN sensor to promote the development of rapid detection technology in the field of pharmaceutical analysis.

\section{Conflict of Interest Statement}

The authors declare that they have no competing interests.

\section{REFERENCES}

1 Lipka E, Vaccher C. Quantitative analysis of drugs in biological matrices by HPLC hyphenated to fluorescence detection. Bioanalysis, 2015,7(6):743-762

2 Toyo'oka T. Diagnostic Approach to disease using noninvasive samples based on derivatization and LC-ESIMS/MS. Biol Pharm Bull, 2016,39,1397-1411

3 Chen XX, Lin ZZ, Hong CY, et al. A dichromatic labelfree aptasensor for sulfadimethoxine detection in fish and water based on AuNPs color and fluorescent dyeing of double-stranded DNA with SYBR Green I. Food Chem, 2020,309:125712

4 Michel BY, Dziuba D, Benhida R, et al. Probing of nucleic acid structures, dynamics, and interactions with evironment-sensitive fluorescent labels. Front Chem, 2020,8:112

5 Jiang LD, Lu R, Ye L. Towards detection of glycoproteins using molecularly imprinted nanoparticles and boronic acid-modified fluorescent probe. Polymers, 2019,11:173

6 Lim GW, Lim JK, Ahmad AL, et al. Fluorescent molecularly imprinted polymer based on Navicula sp. frustules for optical detection of lysozyme. Anal Bioanal Chem, 2016,408:2083-2093

7 Zhu YY, Cui MX, Ma JJ, et al. Fluorescence detection of $\mathrm{D}$-aspartic acid based on thiol-ene cross-linked. Sensor Actuat B-Chem, 2020,305:127323

8 Long Z, Shen S, Lu Y, et al. Monodisperse core-shellstructured $\mathrm{SiO}_{2} @ \mathrm{Gd}_{2} \mathrm{O}_{3}: \mathrm{Eu}^{3+} @ \mathrm{SiO}_{2} @ \mathrm{MIP}$ nanospheres for specific identification and fluorescent determination of carbaryl in green tea molecularly imprinted optical fiber probe. Anal Bioanal Chem, 2019,411:4221-4229

9 Zakery M, Ensafi AA, Rezaei B. A novel optosensor for rapid detection of difenoconazole using molecularly imprinted polymers. IEEE Sensor J, 2018,18(23):94669470

10 Kazemifard N, Ensafi AA, Rezaei B. Green synthesized carbon dots embedded in silica molecularly imprinted polymers, characterization and application as a rapid and selective fluorimetric sensor for determination of thiabendazole in juices. Food Chem, 2020,310:125812

$11 \mathrm{Xu}$ JJ, Miao HH, Wang JX, et al. Molecularly imprinted synthetic antibodies: from chemical design to biomedical applications. Small, 2020:1906644

12 Liu GY, Huang XD, Li LY, et al. Recent advances and perspectives of molecularly imprinted polymer-based fluorescent sensors in food and environment analysis. Nanomaterials, 2019,9:1030

13 Santos H, Martins RO, Soares DA, et al. Molecularly imprinted polymers for miniaturized sample preparation techniques: strategies for chromatographic and mass spectrometry methods. Anal Method, 2020,12:894-911
14 Azizi A, Bottaro CS. A critical review of molecularly imprinted polymers for the analysis of organic pollutants in environmental water samples. J Chromatogr A, 2020,1614:460603

15 Yang Q, Li JH, Wang XY, et al. Strategies of molecular imprinting-based fluorescence sensors for chemical and biological analysis. Biosens Bioelectron, 2018,112:5471

16 Sobiech M, Bujak P, Luliński P, et al. Semiconductor nanocrystal-polymer hybrid nanomaterials and their application in molecular imprinting. Nanoscale, 2019, 11:12030

17 Rao KSVK, Liu HG, Lee Y. Fluorescence spectroscopy of polymer systems doped with rare-earth metal ions and their complexes. Appl Spectrosc Rev, 2010,45(6):409446

18 Xiong L, Fan Y, Zhang F. Research progress on rare earth nanocrystals for in vivo imaging and sensing in near infrared region. Acta Chimica Sinica, 2019,77:12391249

19 Guo SJ, Wang E. Noble metal nanomaterials: controllable synthesis and application in fuel cells and analytical sensors. Nano Today, 2011,6:240-264

20 Yin HQ, Yin XB. Metal-organic frameworks with multiple luminescence emissions: designs and applications. Acc Chem Res, 2020,53(2):485-495

21 Ren X, Chen L. Quantum dot coated with molecularly imprinted polymer as fluorescence probe for detection of cyphenothrin. Biosens Bioelectron, 2015,64:182-188

22 Wei X, Zhou Z, Dai J, et al. Composites of surface imprinting polymer capped Mn-doped $\mathrm{ZnS}$ quantum dots for room-temperature phosphorescence probing of 2,4,5-trichlorophenol. J Lumin, 2014,155:298-304

23 Ren X, Liu H, Chen L. Fluorescent detection of chlorpyrifos using $\mathrm{Mn}$ (II)-doped $\mathrm{ZnS}$ quantum dots coated with a molecularly imprinted polymer. Microchim Acta, 2015,182:193-200

24 Dan L, Wang HF. Mn-doped ZnS quantum dot imbedded two-fragment imprinting silica for enhanced room temperature phosphorescence probing of domoic acid. Anal Chem, 2013,85:4844-4848

25 Chantada-Vázquez MP, Sánchez-González J, PeñaVázquez E, et al. Synthesis and characterization of novel molecularly imprinted polymer-coated Mn-doped $\mathrm{ZnS}$ quantum dots for specific fluorescent recognition of cocaine. Biosens Bioelectron, 2016,75:213-221

26 Chantada-Vázquez MP, Sánchez-González J, PeñaVázquez E, et al. Simple and sensitive molecularly imprinted polymer - Mn-doped $\mathrm{ZnS}$ quantum dots based fluorescence probe for cocaine and metabolites determination in urine. Anal Chem, 2016,88:2734-2741

27 Guo PQ, Yang W, Hu H, et al. Rapid detection of aflatoxin B1 by dummy template molecularly imprinted polymer capped CdTe quantum dots. Anal Bioanal Chem, 2019,411:2607-2617

28 Ke CB, Lu TL, Chen JL. Fluorometric determination of amifostine and alkaline phosphatase on amphiprotic molecularly imprinted silica crosslinked with binary functional silanes and carbon dots. Biosens Bioelectron, 2020,151:111965

29 Shariati R, Rezaei B, Jamei HR, et al. Manufacturing of a sensitive and selective optical sensor based on 
molecularly imprinted polymers and green carbon dots synthesized from cedrus plant for trace analysis of propranolol. Anal Sci, 2019,35:1083-1088

30 Zakery M, Ensafi AA, Rezael B. Detection of theophylline using molecularly imprinted polymers based on thioglycolic acid-modified CdTe quantum dots. J Iran Chem Soc, 2020,17:601-608

31 Lin ZZ, Li WJ, Chen QC, et al. Rapid detection of malachite green in fish with a fluorescence probe of molecularly imprinted polymer. Int J Polym Anal Ch, 2019,24(2):121-131

32 Shariati R, Rezaei B, Jamei HR, et al. Application of coated green source carbon dots with silica molecularly imprinted polymers as a fluorescence probe for selective and sensitive determination of phenobarbital. Talanta, 2019,194:143-149

33 Ran H, Lin ZZ, Yao QH, et al. Ratiometric fluorescence probe of MIPs@CdTe QDs for trace malachite green detection in fish. Anal Bioanal Chem, 2019,411:527544

34 Ganguli AK, Ganguly A, Vaidya S. Microemulsionbased synthesis of nanocrystalline materials. Chem Soc Rev, 2010,39:474-485

35 Geng YY, Guo ML, Tan J, et al. A fluorescent molecularly imprinted polymer using aptamer as afunctional monomer for sensing of kanamycin. Sensor Actuat B: Chem, 2018,268:47-54

36 Li SH, Ma XH, Pang CH, et al. Fluorometric aptasensor for cadmium(II) by using an aptamer-imprinted polymer as the recognition element. Microchim Acta, 2019,186: 823

37 Xiao JS, Chu ZJ, Zuo J, et al. Fluorescent carbon dots: facile synthesis at room temperature and its application for $\mathrm{Fe}^{2+}$ sensing. J Nanopart Res, 2017,19:84

38 Iqbal A, Tian YJ, Wang XD, et al. Carbon dots prepared by solid state method via citric acid and1,10phenanthroline for selective and sensing detection of $\mathrm{Fe}^{2+}$ and $\mathrm{Fe}^{3+}$. Sensor Actuat B: Chem, 2016,237:408415

39 Li DY, Wang SP, Azad F, et al. Single-step synthesis of polychromatic carbon quantum dots for macroscopic detection of $\mathrm{Hg}^{2+}$. Ecotox Environ Safe, 2020,190:110141

40 Tabaraki R, Sadeghinejad N. Microwave assisted synthesis of doped carbon dots and their application as green and simple turn off-on fluorescent sensor for mercury (II) and iodide in environmental samples. Ecotox Environ Safe, 2018,153:101-106

41 Luo LH, Zhang F, Chen CY, et al. Visual simultaneous detection of hepatitis $\mathrm{A}$ and $\mathrm{B}$ viruses based on a multifunctional molecularly imprinted fluorescence sensor. Anal Chem, 2019,91:15748-15756

42 Zhou TC, Halder A, Sun Y. Fluorescent nanosensor based on molecularly imprinted polymers coated on graphene quantum dots for fast detection of antibiotics. Biosens, 2018,8:82

43 Amjadi M, Jalili R. A molecularly imprinted dualemission carbon dot-quantum dot mesoporous hybrid for ratiometric determination of anti-inflammatory drug celecoxib. Spectrochim Acta A, 2018,191,345-351

44 Fang M, Zhou L, Zhang $\mathrm{H}$, et al. A molecularly imprinted polymers/carbon dots-grafted paper sensor for 3-monochloropropane-1,2-diol determination. Food Chem, 2019,274:156-161

$45 \mathrm{Xu} \mathrm{XM}, \mathrm{Xu} \mathrm{GH}$, Wei FD, et al. Carbon dots coated with molecularly imprinted polymers: A facile bioprobe for fluorescent determination of caffeic acid. J Colloid Interf Sci, 2018,529:568-574

46 Ensafi AA, Nasr-Esfahani P, Rezaei B. Synthesis of molecularly imprinted polymer on carbon quantum dots as an optical sensor for selective fluorescent determination of promethazine hydrochloride. Sens Actuat B: Chem, 2018,257:889-896

47 Luo LH, Feng WB, Hu WT, et al. Molecularly imprinted polymer based hybrid structure $\mathrm{SiO}_{2} @$ MPS-CdTe/CdS: a novel fluorescence probe for hepatitis A virus. Method Appl Fluores, 2019,7:015006

48 Yang J, Lin ZZ, Nur AZ, et al. Detection of trace tetracycline in fish via synchronous fluorescence quenching with carbon quantum dots coated with molecularly imprinted silica. Spectrochim Acta A, 2018,190,450-456

49 Shao MY, Yao M, Saeger SD, et al. Carbon quantum dots encapsulated molecularly imprinted fluorescence quenching particles for sensitive detection of zearalenone in corn sample. Toxins, 2018,10:438

50 Hassanzadeh J, Moghadam BR, Sobhani-Nasab A, et al. Specific fluorometric assay for direct determination of amikacin by molecularly imprinting polymer on high fluorescent g-C3N4 quantum dots. Spectrochim Acta A, 2019,214,451-458

51 Yang J, Lin ZZ, Huang ZY. Rapid detection of trace malachite green using a fluorescence probe based on signal amplification through electrostatic self-assembly of CdTe QDs and polystyrene microsphere. Mar Pollut Bull, 2020,151:110812

$52 \mathrm{Fu}$ YX, Jin $\mathrm{H}, \mathrm{Bu}$ XN, et al. Magnetic and fluorescent nanohybrids with surface imprinting silica as a dualfunctional sensing platform for ratiometric fluorescence detection of phycoerythrin. J Mater Chem C, 2019,7: 11483

53 Liu XQ, Wang T, Wang WJ, et al. A tailored molecular imprinting ratiometric fluorescent sensor based on red/ blue carbon dots for ultrasensitive tetracycline detection. J Ind Eng Chem, 2019,72:100-106

54 Wang XY, Yu SM, Liu W, et al. Molecular imprinting based hybrid ratiometric fluorescence sensor for the visual determination of bovine hemoglobin. ACS Sens, 2018,3:378-385

55 Nasibeh SA, Hosseini MRM. Application of ratiometric fluorescence sensorbased microwave-assisted synthesized CdTe quantum dots and mesoporous structured epitopeimprinted polymers for highly efficient determination of tyrosine phosphopeptide. Anal Methods, 2020,12:63

56 Wang JX, Dai JD, Xu YQ, et al. Molecularly imprinted fluorescent test strip for direct, rapid, and visual dopamine detection in tiny amount of biofluid. Small, 2018,15:18039113

57 Yang Q, Li JH, Wang XY, et al. Ternary emission of a blue-, green-, and red-based molecular imprinting fluorescence sensor for the multiplexed and visual detection of bovine hemoglobin. Anal Chem, 2019,91:6561-6568 
58 Yang YK, Chang YY, Guo YY, et al. Fluorometric microplate-based dimethoate assay using $\mathrm{CdSe} / \mathrm{ZnS}$ quantum dots coated with a molecularly imprinted polymer. Microchim Acta, 2019,186:589

59 Tan L, Guo ML, Tan JA, et al. Development of highluminescence perovskite quantum dots coated with molecularly imprinted polymers for pesticide detection by slowly hydrolysing the organosilicon monomers in situ. Sens Actuat B: Chem, 2019,291:226-234

$60 \mathrm{Li}$ XJ, Jiao HF, Shi XZ, et al. Development and application of a novel fluorescent nanosensor based on FeSe quantum dots embedded silica molecularly imprinted polymer for the rapid optosensing of cyfluthrin. Biosens Bioelectron, 2018,90:268-273

61 María PCV, Carolina DBS, Alba FDR, et al. Development and application of molecularly imprinted polymer - Mn-doped ZnS quantum dot fluorescent optosensing for cocaine screening in oral fluid and serum. Talanta, 2018,181:232-238

62 Li HY, Zhao L, Zhou TY, et al. Single-hole hollow molecularly imprinted polymer embedded carbon dot for fast detection of tetracycline in honey. Talanta, 2018,185:542-549

63 Wan YC, Liu YJ, Liu C, et al. Rapid determination of neomycin in biological samples using fluorescent sensor based on quantum dots with doubly selective binding sites. J Pharmaceut Biomed Anal, 2018,154:75-84

64 Piloto AML, Ribeiro DSM, Rodrigues SSM, et al. Label-free quantum dot conjugates for human protein IL-2 based on molecularly imprinted polymers. Sens Actuat B: Chem, 2020,304:127343

65 Jia Z, Luo Y, Wen HY, et al. A Probe for fluorescence detection of the acetylcholinesterase activity based on molecularly imprinted polymers coated carbon dots. Chem Pharm Bull, 2019,67:795-800

66 Saylan Y, Erdem Ö, Ünal S, et al. An alternative medical diagnosis method: Biosensors for virus detection. Biosens, 2019,65:1-22

67 Demetrios P, Denisa M, Konstantinos, T, et al. Obesity-a risk factor for increased COVID-19 prevalence, severity and lethality (Review). Mol med rep, 2020,22:9-19

68 Huang SY, Guo ML, Tan JA, et al. Novel fluorescence sensor based on all-inorganic perovskite quantum dots coated with molecularly imprinted polymers for highly selective and sensitive detection of omethoate. ACS Appl Mater Interfaces, 2018,10:39056-39063

69 Liu Y, Cao N, Gui WY, et al. Nitrogen-doped graphene quantum dots-based fluorescence molecularly imprinted sensor for thiacloprid detection. Talanta, 2018,183:339344

70 Nsibande SA, Forbes PBC. Development of a quantum dot molecularly imprinted polymer sensor for fluorescence detection of atrazine. Luminescence, 2019,34:480-488

71 Liu XY, Liu QR, Kong FF, et al. Molecularly imprinted fluorescent probe based on hydrophobic $\mathrm{CdSe} / \mathrm{ZnS}$ quantum dots for the detection of methamidophos in fruit and vegetables. Adv Polym Technol, 2018,37:17901796

72 Shirani MP, Rezaei B, Ensafi AA. A novel optical sensor based on carbon dots embedded molecularly imprinted silica for selective acetamiprid detection. Spectrochim
Acta A, 2019,210,36-43

73 Liu CX, Zhao JA, Zhang RR, et al. Development and application of fluorescence sensor and test strip based on molecularly imprinted quantum dots for the selective and sensitive detection of propanil in fish and seawater samples. J Hazard Mater, 2020,389:121884

74 Zhang DW, Tang JQ, Liu HL. Rapid determination of lambda-cyhalothrin using a fluorescent probe based on ionic-liquid-sensitized carbon dots coated with molecularly imprinted polymers. Anal Bioanal Chem, 2019,411:5309-5316

$75 \mathrm{Xu}$ SJ, Zou YW, Zhang HQ. Well-defined hydrophilic "turn-on"-type ratiometric fluorescent molecularly imprinted polymer microspheres for direct and highly selective herbicide optosensing in the undiluted pure milks. Talanta, 2020,211:120711

76 Shi T, Tan LJ, Fu HL, et al. Application of molecular imprinting polymer anchored on CdTe quantum dots for the detection of sulfadiazine in seawater. Mar Pollut Bull, 2019,146:591-597

77 Zheng JH, Cheng KK, Yu PF. Simple preparation of fluorescence probe based on CdTe quantum dots combined with MIP for selective detection of sulfadimidine. J Mater Sci Mater El, 2019,30:2117721184

78 Naphat Y, Piyaluk N, Kochaporn C, et al. A nanocomposite optosensor containing carboxylic functionalized multiwall carbon nanotubes and quantum dots incorporated into a molecularly imprinted polymer for highly selective and sensitive detection of ciprofloxacin. Spectrochim Acta A, 2018,201:382-391

79 Habibeh E, Maliheh A, Hamideh A, et al. MIP-capped terbium MOF-76 for the selective fluorometric detection of cefixime after its preconcentration with magnetic graphene oxide. Sens Actuat B: Chem, 2018,275:145154

$80 \mathrm{Hu}$ YF, Li XD, Liu J, et al. One-pot synthesis of a fluorescent molecularly imprinted nanosensor for highly selective detection of sulfapyridine in water. Anal Methods, 2018,10:884

81 Wang ZH, Zhang YF, Zhang B, et al. $\mathrm{Mn}^{2+}$ doped ZnS QDs modified fluorescence sensor based on molecularly imprinted polymer/sol-gel chemistry for detection of serotonin. Talanta, 2018,190:1-8

82 Ensafi AA, Zakery M, Rezaei B. An optical sensor with specific binding sites for the detection of thioridazine hydrochloride based on ZnO-QDs coated with molecularly imprinted polymer. Spectrochim Acta A, 2019,206:460-465

83 Yang Q, Li CY, Li JH, et al. Rational construction of a triple emission molecular imprinting sensor for accurate nakedeye detection of folic acid. Nanoscale, 2020,12:6529-6536

84 Gui WY, Wang H, Liu Y, et al. Ratiometric fluorescent sensor with molecularly imprinted mesoporous microspheres for malachite green detection. Sens Actuat B: Chem, 2018,266:685-691

$85 \mathrm{Wu} \mathrm{L}$, Lin ZZ, Zeng J, et al. Detection of malachite green in fish based on magnetic fluorescent probe of CdTe QDs/nano-Fe $\mathrm{O}_{4} @$ MIPs. Spectrochim Acta A, 2018,196:117-122

86 Sun CH, Pan LL, Zhang L, et al. A biomimetic fluorescent 
nanosensor based on imprinted polymers modified with carbon dots for sensitive detection of alpha-fetoprotein in clinical samples. Analyst, 2019,144:6760-6772

87 Fang MY, Zhuo K, Chen YJ, et al. Fluorescent probe based on carbon dots/silica/molecularly imprinted polymer for lysozyme detection and cell imaging. Anal Bioanal Chem, 2019,411:5799-5807

88 Piloto AM, Ribeiro DSM, Rodrigues SSM, et al. Plastic antibodies tailored on quantum dots for an optical detection of myoglobin down to the femtomolar range. Sci Rep, 2018,8:4944

89 Tan JA, Guo ML, Tan L, et al. Highly efficient fluorescent QDs sensor for specific detection of protein through double recognition of hybrid aptamermolecular imprinted polymers. Sens Actuat B: Chem, 2018,274:627-635

90 Zhang XM, Qin YP, Ye HL, et al. Silicon nanoparticles coated with an epitope-imprinted polymer for fluorometric determination of cytochrome c. Microchim Acta, 2018,185:173

$91 \mathrm{Lu} \mathrm{HZ}, \mathrm{Yu} \mathrm{CW}, \mathrm{Xu}$ SF. A dual reference ion-imprinted ratiometric fluorescence probe for simultaneous detection of silver (I) and lead (II). Sens Actuat B:
Chem, 2019,288:691-698

92 Chmangui A, Driss MR, Touil S, et al. Aflatoxins screening in non-dairy beverages by Mn-doped $\mathrm{ZnS}$ quantum dots-Molecularly imprinted polymer fluorescent probe. Talanta, 2019,199:65-71

93 Zheng L, Zheng YH, Liu Y, et al. Core-shell quantum dots coated with molecularly imprinted polymer for selective photoluminescence sensing of perfluorooctanoic acid. Talanta, 2019,194:1-6

94 Kim D, Lee B. Fluorescence detection of bisphenol A in aqueous solution using magnetite core-shell material with gold nanoclusters prepared by molecular imprinting technique. Korean J Chem Eng, 2019,36(9):1509-1517

95 Üzek R, Sari E, Şenel S, et al. A nitrocellulose paper strip for fluorometric determination of bisphenol A using molecularly imprinted nanoparticles. Microchim Acta, 2019,186:218

96 Zhang X, Yang S, Chen WJ, et al. Magnetic fluorescence molecularly imprinted polymer based on $\mathrm{FeO}_{\mathrm{x}} /$ $\mathrm{ZnS}$ nanocomposites for highly selective sensing of bisphenol A. Polymers, 2019,11:1210

(Received Mar. 24, 2020; revised May 22, 2020) 